\title{
Vegetarian diets and blood pressure among white subjects: results from the Adventist Health Study-2 (AHS-2)
}

\author{
Betty J Pettersen ${ }^{1}+$, Ramtin Anousheh ${ }^{1}$, Jing Fan ${ }^{1}$, Karen Jaceldo-Siegl ${ }^{2}$ and \\ Gary E Fraser ${ }^{1, *}$ \\ 'Department of Epidemiology and Biostatistics, School of Public Health, Loma Linda University, 24785 Stewart \\ Street, Loma Linda, CA 92350, USA: ${ }^{2}$ Department of Nutrition, School of Public Health, Loma Linda University, \\ Loma Linda, CA, USA
}

Submitted 19 May 2011: Accepted 24 November 2011: First published online 10 January 2012

\begin{abstract}
Objective: Previous work studying vegetarians has often found that they have lower blood pressure (BP). Reasons may include their lower BMI and higher intake levels of fruit and vegetables. Here we seek to extend this evidence in a geographically diverse population containing vegans, lacto-ovo vegetarians and omnivores.

Design: Data are analysed from a calibration sub-study of the Adventist Health Study-2 (AHS-2) cohort who attended clinics and provided validated FFQ. Criteria were established for vegan, lacto-ovo vegetarian, partial vegetarian and omnivorous dietary patterns.

Setting: Clinics were conducted at churches across the USA and Canada. Dietary data were gathered by mailed questionnaire.

Subjects: Five hundred white subjects representing the AHS-2 cohort.

Results: Covariate-adjusted regression analyses demonstrated that the vegan vegetarians had lower systolic and diastolic BP $(\mathrm{mmHg})$ than omnivorous Adventists $(\beta=-6 \cdot 8$, $P<0.05$ and $\beta=-6.9, P<0 \cdot 001)$. Findings for lacto-ovo vegetarians $(\beta=-9 \cdot 1$, $P<0 \cdot 001$ and $\beta=-5 \cdot 8, P<0 \cdot 001$ ) were similar. The vegetarians (mainly the vegans) were also less likely to be using antihypertensive medications. Defining hypertension as systolic $\mathrm{BP}>139 \mathrm{mmHg}$ or diastolic $\mathrm{BP}>89 \mathrm{mmHg}$ or use of antihypertensive medications, the odds ratio of hypertension compared with omnivores was $0.37(95 \% \mathrm{CI} 0 \cdot 19,0 \cdot 74), 0.57(95 \% \mathrm{CI} 0 \cdot 36,0 \cdot 92)$ and $0.92(95 \% \mathrm{CI}$ $0 \cdot 50,1 \cdot 70)$, respectively, for vegans, lacto-ovo vegetarians and partial vegetarians. Effects were reduced after adjustment for BMI.

Conclusions: We conclude from this relatively large study that vegetarians, especially vegans, with otherwise diverse characteristics but stable diets, do have lower systolic and diastolic BP and less hypertension than omnivores. This is only partly due to their lower body mass.
\end{abstract}

Keywords Vegetarian diet Blood pressure
Elevated blood pressure (BP) is a modifiable major risk factor for cardiovascular morbidity. Estimates are that suboptimal BP (systolic BP $>115 \mathrm{mmHg}$ ) is responsible for $62 \%$ of cerebrovascular diseases and $49 \%$ of IHD, with little variation by $\operatorname{sex}^{(1)}$. The National Center for Health Statistics estimates an overall prevalence of hypertension (systolic $\mathrm{BP} \geq 140 \mathrm{mmHg}$ or diastolic $\mathrm{BP} \geq 90 \mathrm{mmHg}$ or taking medications for hypertension) in 2003-2006 of $31 \cdot 3 \%^{(2)}$, and of $47 \cdot 3 \%$ in those aged 55 years or more ${ }^{(3)}$. This suggests that 50 million or more Americans have high BP. Favourable BP levels are associated with greater

$\uparrow$ Present address: Department of Community Medicine, Faculty of Medicine, University of Troms $\varnothing$, NO-9037 Troms $\varnothing$, Norway. probability of survival to age 85 years as well as increased longevity without co-morbidity ${ }^{(4)}$.

The differences in prevalence rates for hypertension between certain populations and age groups have been partially explained by differences in intakes of particular nutrients, although the evidence for some is weak. These include positive associations with $\mathrm{Na}$, alcohol and total protein, and negative associations with $\mathrm{K}, \mathrm{Ca}$ and $\mathrm{Mg}^{(5,6)}$. Recent work suggests that a diet similar to the Dietary Approaches to Stop Hypertension (DASH) diet, but containing more lean red meat, is still superior to a more typical diet ${ }^{(7)}$ in postmenopausal women, but results of feeding studies of meat on BP are inconsistent ${ }^{(8,9)}$. Dietary fats have been examined, with some studies showing no 
convincing effects on BP, while other studies indicate a possible relationship depending on fat type ${ }^{(10-15)}$. Fibre has also been studied, and recent meta-analyses of trials have shown small but significant effects mainly on diastolic BP and more pronounced effects in hypertensive individuals ${ }^{(16,17)}$.

The effects of a vegetarian diet on BP have been evaluated by several previous studies over three decades. Most of these have been small short-term feeding studies $^{(9,18-20)}$ or cross-sectional comparisons also of relatively small selected study groups of vegetarians and others $^{(21-24)}$. Melby et al. have studied this association in African Americans ${ }^{(25,26)}$ with mixed results. Most of these studied the lacto-ovo vegetarian rather than vegan diet. An exception to limitations of size and diversity of subjects is a very large cross-sectional comparison of British vegetarians (vegans and lacto-ovo vegetarians) and health-conscious non-vegetarians ${ }^{(27)}$, where significant effects were found, mainly in the vegans.

In the current paper we present results of a crosssectional comparison within a relatively large and diverse group where the vegetarian and non-vegetarian habits were generally long term, and where vegetarians were divided to vegans and non-vegans. This study group is representative of a national cohort of Seventh-day Adventists ${ }^{(28)}$ which spans a wide range of socio-economic status, age and geographic location, and both genders. Alcohol consumption is minimal or absent.

\section{Materials and methods}

The Adventist Health Study-2 (AHS-2) is a cohort study of 96000 Adventists living in the USA and Canada who enrolled between 2002 and $2007^{(28)}$. We analysed data from the AHS-2 calibration study which was designed mainly to evaluate the accuracy of collection and reporting of data. The study subjects are a representative sample of 504 non-black individuals (about 3\% Asian), randomly selected from the parent cohort by church, and then subject-within-church. Recruitment and enrolment methods of the parent cohort and calibration study are detailed elsewhere ${ }^{(28)}$.

Relevant data were gathered from a self-administered FFQ and a $7 \mathrm{~d}$ physical activity recall. In addition, BP and BMI were measured and overnight urine collected during a brief clinical examination performed by a travelling team of trained technicians ${ }^{(29)}$.

Calibration study subjects came to the clinic fasting, having been instructed to take their usual medications. They were instructed to empty their bladder and were then seated in a quiet room at a comfortable temperature for approximately $10 \mathrm{~min}$ before their systolic and diastolic BP were measured using an Omron automated sphygmomanometer ${ }^{(30)}$. Three readings were taken $5 \mathrm{~min}$ apart. The mean of the second and third readings was
Table 1 The twenty-five items from the FFQ used for classification of dietary patterns

Red meat ( 3 items)
Hamburger, ground beef
Processed beef, lamb
Beef or lamb a main dish
Poultry ( 2 items)
Processed chicken or turkey
Chicken or turkey
Fish (4 items)
White fish
Salmon
Canned tuna, tuna salad, etc.
Other fish
Dairy products (15 items)
Cottage cheese
Cream cheese, cheese spread
American processed, cheddar cheese
Low-fat cheese, mozzarella, ricotta
Butter
Milk (whole or $2 \%)$
Low-fat milk
Evaporated milk
Low-fat yoghurt
Regular yoghurt
Other dairy products
Ice cream, milk shakes
Ice milk, frozen yoghurt
Meal replacement drink
Hot chocolate
Eggs ( 1 item)

used for the present analyses unless there was a difference of more than $5 \mathrm{mmHg}$, in which case we used the mean of all three readings. We excluded four subjects with a mean recorded systolic BP of less than $80 \mathrm{mmHg}$, leaving 500 subjects for analysis.

Dietary pattern was determined using information from the FFQ about intake of twenty-five different food items (Table 1) relevant to vegetarian status. Participants were asked to report their usual or average diet during the past year. Each food item allowed up to nine frequency response options, ranging from 'never or rarely' to ' $6+$ times/d'. The validity of this FFQ is relatively high for most nutrients and foods when compared with the means of the six dietary recalls ${ }^{(31,32)}$. Frequencies of intake were converted to daily equivalents and these were used to construct composite food variables that measured intakes of red meat, poultry, fish, eggs and dairy foods. Values of these variables allowed subjects to be assigned to a dietary pattern as shown in Table 2 .

There were small amounts of missing data in the dietary variables. For about half of these variables a random $10 \%$ subset of initially missing data was later filled-in by telephone. We used this to guide imputation ${ }^{(33)}$ of the remaining missing data for these variables. For other variables we assumed that the data were missing at random, which even if not quite correct will cause little bias when the missing data rate is small ${ }^{(33)}$. Imputation was performed at the level of the composite variables meat, fish, dairy and eggs, and was also conditional on other covariates and the dependent variable in any 
Table 2 Definitions of dietary patterns

\begin{tabular}{ll}
\hline Main dietary pattern & Definition \\
\hline Vegan & $\begin{array}{c}\text { Eat meat, fish and dairy less than once } \\
\text { monthly } \\
\text { Eat meat and/or fish less than once monthly, } \\
\text { and dairy more than once monthly } \\
\text { Includes pesco-vegetarians, who eat meat } \\
\text { less than once monthly and fish at least } \\
\text { once monthly, and semi-vegetarians, } \\
\text { who eat meat at least once monthly and } \\
\text { fish and meat less than once weekly }\end{array}$ \\
Non-vegetarians & $\begin{array}{l}\text { fo specific dietary restrictions as to } \\
\text { frequencies of meat, fish and dairy }\end{array}$ \\
\hline
\end{tabular}

particular statistical model. The imputation software used was the Hmisc package for $\mathrm{R}$ version $2 \cdot 6 \cdot 0$ (R Foundation for Statistical Computing, Vienna, Austria) ${ }^{(34)}$.

Physical activity was measured using a detailed hourby-hour telephone recall about type, intensity and duration of different physical activities during the preceding week $^{(35)}$. These activities were summed to produce hours per week of moderate, hard or very hard exercise (metabolic equivalent task levels $24 \cdot 5$ ).

Information on medication use in the present study was obtained along with the $24 \mathrm{~h}$ recalls. A cardiologist subsequently identified all medications used for treatment of or known to reduce BP. The use of such medications is reported as none or some. This population largely abstains from alcohol use $(6.75 \%$ admit to current use, mostly infrequent), although it was tested as a covariate.

The study was approved by the institutional review board of Loma Linda University and all subjects gave written informed consent.

\section{Statistical analysis}

ANOVA was performed to test null hypotheses of equal means between dietary groups for continuous variables, while the $\chi^{2}$ test was used for categorical variables. These were performed using the SAS statistical software package release 9.2 (SAS Institute Inc., Cary, NC, USA). Linear and logistic regression modelling was performed using statistical software R version $2 \cdot 10 \cdot 1$ (R Foundation for Statisical Computing). Where required, hypotheses involving interactions were tested using product terms in regression models.

\section{Results}

\section{Demographics}

The four groups of participants are presented in Table 3. Ten per cent were vegan, $36 \%$ were lacto-ovo vegetarian, $14 \%$ partial vegetarian and $40 \%$ non-vegetarian (Table 3 ).

The age distribution was significantly different between the groups with the vegans being older. The average age of the participants was 62.7 years and $64 \%$ were women.
There was a large range of educational attainment and subjects lived in all major regions of the USA and Canada. More of the vegetarians had at least 50 years of church membership. The following percentages of the study population came from the stated regions: West $23 \cdot 8 \%$; Northwest 16.2\%; Mountain 4.0\%; Midwest 18.2\%; Mid and North East 11.5\%; South 21.6\%; Canada $4.6 \%$. The proportions in the various dietary categories did not vary significantly by region although there was a tendency towards a higher proportion of vegetarians in the Northwest and Mountain regions.

\section{Blood pressure and bypertension medication}

The mean BP levels were relatively low for all subjects (systolic $125 \cdot 2 \mathrm{mmHg}$, diastolic $75 \cdot 2 \mathrm{mmHg}$ ), moderately higher for those treated (systolic $133.3 \mathrm{mmHg}$, diastolic $76.3 \mathrm{mmHg}$ ) and lowest in untreated subjects (systolic $122.5 \mathrm{mmHg}$, diastolic $74.8 \mathrm{mmHg}$ ). The proportion taking some medication known to reduce BP was for the whole population $25 \cdot 2 \% ; 22.8 \%$ for women and $29 \cdot 4 \%$ for men. We found no significant differences in BP between the four dietary groups among those taking antihypertensive medication.

\section{Blood pressure, age, gender and physical activity}

There were no significant differences in BP by gender. As expected there were strong age effects on systolic $\mathrm{BP}$, but these were not seen for diastolic BP. We could not demonstrate significant effects of physical activity on BP.

\section{Relationship between diet and blood pressure}

Adjusting for age and gender (Table 4) and including only non-treated individuals, systolic BP was significantly lower in vegans and lacto-ovo vegetarians $(\beta=-6 \cdot 8$ $\mathrm{mmHg}, P<0.05$ and $\beta=-9.1 \mathrm{mmHg}, P<0.001)$ when compared with non-vegetarians, and results were broadly similar for diastolic BP $(\beta=-6.9 \mathrm{mmHg}, P<0.001$ and $\beta=-5.8 \mathrm{mmHg}, P<0 \cdot 001$; Table 4$)$. There was a significantly higher systolic BP at older ages $(0.6 \mathrm{mmHg}$ higher per year, $P<0 \cdot 001)$ and there was no discernible effect of physical activity.

BMI was significantly associated with both systolic and diastolic BP (Table 5). Where normal BMI was the reference, overweight subjects $\left(\mathrm{BMI}=25 \cdot 0-29 \cdot 9 \mathrm{~kg} / \mathrm{m}^{2}\right)$ had systolic BP $3.1 \mathrm{mmHg}$ higher (NS) and obese subjects $\left(\mathrm{BMI} \geq 30 \cdot 0 \mathrm{~kg} / \mathrm{m}^{2}\right.$ ) on average had systolic BP $11.9 \mathrm{mmHg}$ higher $(P<0 \cdot 001)$. For diastolic BP these statistics were respectively $3.5 \mathrm{mmHg}(P<0.01)$ and $9.6 \mathrm{mmHg}(P<0 \cdot 001$; Table 5$)$. Creating 2-unit wide BMI categories between BMI of $<18 \mathrm{~kg} / \mathrm{m}^{2}$ and $>32 \mathrm{~kg} / \mathrm{m}^{2}$ showed a linear association with BP from lowest to highest categories for both systolic BP and diastolic BP.

When adjusting for BMI the same trends with dietary pattern observed in Table 4 were seen, although the differences were less (Table 5). Clearly BMI is to some extent an 
Table 3 Main characteristics of the study population: white subjects representing the Adventist Health Study-2 (AHS-2) cohort, USA and Canada

\begin{tabular}{|c|c|c|c|c|c|c|c|c|c|}
\hline & \multicolumn{2}{|c|}{ Vegan $(n 49 ; 10 \%)$} & \multicolumn{2}{|c|}{ Lacto-ovo vegetarian ( $n$ 184; $36 \%)$} & \multicolumn{2}{|c|}{ Partial vegetarian $(n 69 ; 14 \%)$} & \multicolumn{2}{|c|}{ Non-vegetarian ( $n$ 198; $40 \%)$} & \multirow{2}{*}{$\frac{\text { All }(n 500)}{P \text { value }}$} \\
\hline & $n$ or Mean & $\%$ or SD & $n$ or Mean & $\%$ or SD & $n$ or Mean & $\%$ or SD & $n$ or Mean & $\%$ or SD & \\
\hline \multicolumn{10}{|l|}{ Age (years) } \\
\hline $30-39$ & 0 & 0 & 5 & 3 & 3 & 4 & 8 & 4 & $0.03 \|$ \\
\hline $40-49$ & 1 & 2 & 33 & 18 & 11 & 16 & 43 & 22 & \\
\hline $50-59$ & 13 & 27 & 46 & 25 & 18 & 26 & 44 & 22 & \\
\hline $60-69$ & 13 & 27 & 33 & 18 & 21 & 31 & 48 & 24 & \\
\hline$\geq 70$ & 22 & 44 & 67 & 36 & 16 & 23 & 55 & 28 & \\
\hline Mean and SD & $67 \cdot 6$ & $11 \cdot 6$ & $63 \cdot 5$ & $14 \cdot 9$ & $61 \cdot 4$ & $12 \cdot 6$ & $61 \cdot 0$ & $13 \cdot 2$ & 0.019 \\
\hline \multicolumn{10}{|l|}{ Gender } \\
\hline Women & 35 & 71 & 116 & 63 & 47 & 68 & 122 & 62 & $0.52 \|$ \\
\hline \multicolumn{10}{|l|}{ Education } \\
\hline High school or below & 16 & 33 & 34 & 19 & 14 & 21 & 39 & 20 & $0 \cdot 28 \|$ \\
\hline Some college & 16 & 33 & 68 & 38 & 22 & 33 & 82 & 43 & \\
\hline Bachelors and above & 16 & 33 & 77 & 43 & 30 & 46 & 70 & 37 & \\
\hline \multicolumn{10}{|l|}{ BMlt $\left(\mathrm{kg} / \mathrm{m}^{2}\right)$} \\
\hline$\leq 24.9$ & 35 & 72 & 109 & 59 & 32 & 46 & 53 & 27 & $<0.0001 \|$ \\
\hline $25 \cdot 0-29 \cdot 9$ & 8 & 16 & 52 & 28 & 22 & 32 & 69 & 35 & \\
\hline$\geq 30 \cdot 0$ & 6 & 12 & 23 & 13 & 15 & 22 & 76 & 38 & \\
\hline Mean and SD & $24 \cdot 0$ & $5 \cdot 9$ & $25 \cdot 1$ & $5 \cdot 2$ & $26 \cdot 3$ & $5 \cdot 2$ & $29 \cdot 5$ & $6 \cdot 6$ & $<0.0001$ \\
\hline \multicolumn{10}{|l|}{$\mathrm{BP}$ (range) (mmHg) } \\
\hline All & $(n 49)$ & & $(n$ 184) & & $(n 69)$ & & $(n$ 198) & & \\
\hline Systolic (83.0-199·0), mean and SD & $123 \cdot 5$ & $21 \cdot 6$ & $123 \cdot 2$ & $20 \cdot 8$ & $124 \cdot 4$ & $19 \cdot 5$ & $127 \cdot 9$ & $18 \cdot 2$ & $0 \cdot 11$ ब \\
\hline Diastolic (48.5-109.0), mean and SD & $71 \cdot 3$ & $8 \cdot 8$ & $73 \cdot 0$ & $9 \cdot 6$ & $75 \cdot 4$ & $9 \cdot 6$ & $78 \cdot 1$ & $9 \cdot 6$ & $<0.00019$ \\
\hline Untreated & $(n 44)$ & & $(n$ 139) & & $(n 50)$ & & $(n$ 141) & & \\
\hline Systolic (83.0-184.5), mean and SD & $123 \cdot 0$ & $22 \cdot 4$ & $119 \cdot 3$ & $19 \cdot 1$ & $121 \cdot 1$ & $19 \cdot 1$ & $126 \cdot 1$ & $17 \cdot 3$ & 0.03 \\
\hline Diastolic (48.5-109.0), mean and SD & $70 \cdot 9$ & $9 \cdot 1$ & $72 \cdot 6$ & $19 \cdot 1$ & $74 \cdot 2$ & $8 \cdot 6$ & $78 \cdot 3$ & $9 \cdot 7$ & $<0.00019$ \\
\hline Treated & $(n 5)$ & & $(n 45)$ & & $(n 19)$ & & $(n 57)$ & & \\
\hline Systolic (90·3-199·0), mean and SD & $127 \cdot 6$ & $12 \cdot 1$ & $135 \cdot 4$ & $21 \cdot 3$ & $132 \cdot 9$ & $18 \cdot 2$ & $132 \cdot 4$ & $19 \cdot 8$ & $0 \cdot 80$ ฯ \\
\hline Diastolic $(54 \cdot 5-100 \cdot 5)$, mean and SD & $74 \cdot 7$ & $5 \cdot 2$ & $74 \cdot 0$ & $9 \cdot 5$ & $78 \cdot 5$ & $11 \cdot 6$ & $77 \cdot 5$ & $9 \cdot 1$ & 0.219 \\
\hline \multicolumn{10}{|l|}{ BP medication } \\
\hline No & 44 & 90 & 139 & 76 & 50 & 72 & 141 & 71 & $0.06 \|$ \\
\hline Some & 5 & 10 & 45 & 24 & 19 & 25 & 57 & 29 & \\
\hline Women & 3 & 60 & 26 & 58 & 12 & 63 & 32 & 56 & $0.96 \|$ \\
\hline Men & 2 & 40 & 19 & 42 & 7 & 37 & 25 & 44 & \\
\hline \multicolumn{10}{|l|}{ Duration of church membership§ } \\
\hline$<25$ years & 6 & 13 & 14 & 8 & 11 & 16 & 39 & 21 & $0.0044 \|$ \\
\hline $25-50$ years & 10 & 21 & 51 & 29 & 27 & 40 & 63 & 33 & \\
\hline $50-75$ years & 23 & 49 & 92 & 51 & 24 & 36 & 70 & 37 & \\
\hline$\geq 75$ years & 8 & 17 & 22 & 12 & 5 & 8 & 16 & 9 & \\
\hline
\end{tabular}

BP, blood pressure.

Data are presented as numbers and percentages or means and standard deviations where appropriate, with significance tests $(n 500)$.

+13 missing BMI data.

‡Mean of last two BP measurements if difference between last two measurements is $\leq 5 \mathrm{mmHg}$; mean of three BP measurements if difference between last two measurements is $>5 \mathrm{mmHg}$.

$\$ 19$ missing data on age at baptism.

$\| P$ value for $\chi^{2}$ test for dependency among dietary patterns.

$\uparrow P$ value for ANOVA test of equality of means among dietary patterns. 
Table 4 Parameter estimates ( $\beta$ coefficient, 95\% confidence interval) relating blood pressure (BP) and dietary pattern by antihypertensive medication status, adjusted for gender and age: white subjects representing the Adventist Health Study-2 (AHS-2) cohort, USA and Canada

\begin{tabular}{|c|c|c|c|c|c|c|c|c|}
\hline & \multicolumn{4}{|c|}{ Systolic BP } & \multicolumn{4}{|c|}{ Diastolic BP } \\
\hline & \multicolumn{2}{|c|}{ No treatment ( $n$ 374) } & \multicolumn{2}{|c|}{ All $(n 500)$} & \multicolumn{2}{|c|}{ No treatment ( $n$ 374) } & \multicolumn{2}{|c|}{ All $(n 500)$} \\
\hline & $\beta$ & $95 \% \mathrm{Cl}$ & $\beta$ & $95 \% \mathrm{Cl}$ & $\beta$ & $95 \% \mathrm{Cl}$ & $\beta$ & $95 \% \mathrm{Cl}$ \\
\hline Non-vegetarian & 0.0 & & $0 \cdot 0$ & & 0.0 & & 0.0 & \\
\hline Partial vegetarian & $-5 \cdot 1$ & $-10 \cdot 6,0 \cdot 4$ & $-4 \cdot 9$ & $-10 \cdot 5,0 \cdot 8$ & $-4 \cdot 4^{\star \star}$ & $-7 \cdot 5,-1 \cdot 2$ & $-4 \cdot 2^{\star \star}$ & $-7 \cdot 3,-1 \cdot 07$ \\
\hline Lacto-ovo vegetarian & $-9 \cdot 1^{\star \star \star}$ & $-13 \cdot 1,-4 \cdot 9$ & $-8 \cdot 9^{\star \star \star}$ & $-13 \cdot 1,-4 \cdot 7$ & $-5 \cdot 8^{\star \star *}$ & $-8 \cdot 1,-3 \cdot 4$ & $-5 \cdot 7^{\star \star \star}$ & $-7 \cdot 5,-2 \cdot 9$ \\
\hline Vegan & $-6 \cdot 8^{*}$ & $-12 \cdot 7,-1 \cdot 0$ & $-7 \cdot 1^{\star *}$ & $-12 \cdot 9,-1 \cdot 4$ & $-6 \cdot 9^{\star \star *}$ & $-10 \cdot 1,-3 \cdot 7$ & $-6 \cdot 6^{\star \star \star}$ & $-9 \cdot 0,-3 \cdot 1$ \\
\hline Gender (female/male) & $0 \cdot 7$ & $-3 \cdot 0,4 \cdot 5$ & $2 \cdot 5$ & $-0.8,5.9$ & $-1 \cdot 2$ & $-3 \cdot 3,1 \cdot 0$ & $-0 \cdot 8$ & $-2 \cdot 6,1 \cdot 0$ \\
\hline Age (per year) & $0 \cdot 6^{\star \star \star}$ & $0 \cdot 5,0 \cdot 8$ & $0 \cdot 6^{\star \star *}$ & $0 \cdot 5,0 \cdot 8$ & -0.003 & $-0.08,0.08$ & -0.04 & $-0 \cdot 1,0 \cdot 02$ \\
\hline Medication (no/yes) & \multicolumn{2}{|c|}{ No entries } & 0.6 & $-4 \cdot 8,6 \cdot 1$ & \multicolumn{2}{|c|}{ No entries } & 0.57 & $-3 \cdot 5,2 \cdot 4$ \\
\hline Exerciset & -0.01 & $-0 \cdot 3,0 \cdot 3$ & 0.04 & $-0 \cdot 2,0 \cdot 3$ & -0.05 & $-0 \cdot 2,0 \cdot 1$ & -0.03 & $-0 \cdot 2,0 \cdot 1$ \\
\hline Lacto-ovo $\times$ medication & \multicolumn{2}{|c|}{ No entries } & $9 \cdot 5^{*}$ & $1 \cdot 5,17 \cdot 6$ & \multicolumn{2}{|c|}{ No entries } & \multicolumn{2}{|c|}{ No entries } \\
\hline
\end{tabular}

${ }^{*} P<0.05 ;{ }^{* *} P<0.01 ;{ }^{* * *} P<0.001$

tHours per week of moderate/hard/very hard physical activity.

Table 5 Parameter estimates ( $\beta$ coefficient, $95 \%$ confidence interval) relating blood pressure (BP) and dietary pattern by antihypertensive medication status, adjusted for BMI, age and gender: white subjects representing the Adventist Health Study-2 (AHS-2) cohort, USA and Canada

\begin{tabular}{|c|c|c|c|c|c|c|c|c|}
\hline & \multicolumn{4}{|c|}{ Systolic BP } & \multicolumn{4}{|c|}{ Diastolic BP } \\
\hline & \multicolumn{2}{|c|}{ No treatment (n 374) } & \multicolumn{2}{|c|}{ All $(n 500)$} & \multicolumn{2}{|c|}{ No treatment ( $n$ 374) } & \multicolumn{2}{|c|}{ All $(n 500)$} \\
\hline & $\beta$ & $95 \% \mathrm{Cl}$ & $\beta$ & $95 \% \mathrm{Cl}$ & $\beta$ & $95 \% \mathrm{Cl}$ & $\beta$ & $95 \% \mathrm{Cl}$ \\
\hline Non-vegetarian & 0.0 & & 0.0 & & 0.0 & & 0.0 & \\
\hline Partial vegetarian & $-2 \cdot 8$ & $-8 \cdot 3,2 \cdot 6$ & $-3 \cdot 4$ & $-6 \cdot 4,3 \cdot 0$ & $-2 \cdot 6$ & $-5 \cdot 5,0 \cdot 4$ & $-2 \cdot 7$ & $-5 \cdot 6,0 \cdot 2$ \\
\hline Lacto-ovo vegetarian & $-5 \cdot 9^{\star \star}$ & $-9 \cdot 7,-1 \cdot 5$ & $-6 \cdot 0^{\star \star}$ & $-9 \cdot 4,-1 \cdot 2$ & $-3 \cdot 2^{\star \star}$ & $-5.4,-0.9$ & $-3 \cdot 0^{\star \star}$ & $-5 \cdot 3,-0 \cdot 8$ \\
\hline Vegan & $-3 \cdot 8$ & $-10 \cdot 7,1 \cdot 2$ & $-4 \cdot 3$ & $-11 \cdot 1,0 \cdot 2$ & $-4 \cdot 2^{\star \star}$ & $-7 \cdot 9,-1 \cdot 4$ & $-4 \cdot 3^{*}$ & $-7 \cdot 3,-0 \cdot 3$ \\
\hline \multicolumn{9}{|l|}{ BMl $\left(\mathrm{kg} / \mathrm{m}^{2}\right)$} \\
\hline$\leq 24.9$ & $0 \cdot 0$ & & $0 \cdot 0$ & & $0 \cdot 0$ & & $0 \cdot 0$ & \\
\hline $25 \cdot 0-29 \cdot 9$ & $3 \cdot 1$ & $-1 \cdot 1,7 \cdot 0$ & 2.9 & $-0.8,6.5$ & $3 \cdot 5^{\star \star}$ & $1 \cdot 3,5 \cdot 6$ & $3 \cdot 8^{\star \star \star}$ & $1 \cdot 9,5 \cdot 7$ \\
\hline$\geq 30 \cdot 0$ & $11 \cdot 9^{\star \star \star}$ & $7 \cdot 1,16 \cdot 4$ & $10 \cdot 8^{\star \star \star}$ & $6 \cdot 8,14 \cdot 9$ & $9 \cdot 6^{\star \star \star}$ & $7 \cdot 1,12 \cdot 1$ & $9 \cdot 5^{\star \star \star}$ & $7 \cdot 4,11 \cdot 6$ \\
\hline Gender (female/male) & 0.7 & $-3 \cdot 0,4 \cdot 3$ & $2 \cdot 5$ & $-0 \cdot 8,4 \cdot 0$ & $-1 \cdot 3$ & $-3 \cdot 3,0 \cdot 7$ & -0.6 & $-2 \cdot 4,1 \cdot 1$ \\
\hline Age (per year) & $0 \cdot 7^{\star \star \star}$ & $0.5,0.8$ & $0 \cdot 7^{\star \star \star}$ & $0.5,0.8$ & 0.003 & $-0.07,0.07$ & -0.02 & $-0.1,0.04$ \\
\hline Medication (no/yes) & \multicolumn{2}{|c|}{ No entries } & $-1 \cdot 4$ & $-6 \cdot 8,4 \cdot 0$ & \multicolumn{2}{|c|}{ No entries } & $-2 \cdot 3$ & $-2 \cdot 1,0.5$ \\
\hline Exerciset & $0 \cdot 1$ & $-0.2,0.4$ & $0 \cdot 1$ & $-0 \cdot 1,0 \cdot 4$ & 0.004 & $-0 \cdot 1,0 \cdot 1$ & 0.01 & $-0 \cdot 1,0 \cdot 1$ \\
\hline Lacto-ovo $\times$ medication & \multicolumn{2}{|c|}{ No entries } & $10 \cdot 3^{*}$ & $2 \cdot 3,18 \cdot 3$ & \multicolumn{2}{|c|}{ No entries } & \multicolumn{2}{|c|}{ No entries } \\
\hline
\end{tabular}

${ }^{*} P<0.05 ;{ }^{\star *} P<0.01 ;{ }^{* \star *} P<0.001$.

tHours per week of moderate/hard/very hard physical activity.

intermediary between diet and BP effect. However, significant dietary effects were still seen when the analyses were confined to the 224 subjects with $\mathrm{BMI}<25 \cdot 0 \mathrm{~kg} / \mathrm{m}^{2}$. Specifically, with non-vegetarians as the reference, vegans had lower systolic BP by $7 \cdot 12 \mathrm{mmHg}(P=0 \cdot 06)$, lacto-ovo vegetarians were lower by $5.55 \mathrm{mmHg}(P=0.06)$ and partial vegetarians by $2.75 \mathrm{mmHg}(P=0 \cdot 47)$. For diastolic BP vegans were lower by $5 \cdot 10 \mathrm{mmHg}(P=0 \cdot 006)$, lacto-ovo vegetarians were lower by $3.07 \mathrm{mmHg}(P=0.03)$ and partial vegetarians lower by $0.52 \mathrm{mmHg}(P=0.78)$. When in addition adjusting for BMI these effects in nonoverweight vegetarians diminished by $1-2 \mathrm{mmHg}$ for systolic BP (test for dietary effects: $P=0.17$ for vegans, $P=0.11$ for lacto-ovo vegetarians) and by about $1 \mathrm{mmHg}$ for diastolic BP (test for dietary effects: $P=0.02$ for vegans, $P=0.06$ lacto-ovo vegetarians).

Defining hypertension as average systolic $\mathrm{BP}>139 \mathrm{mmHg}$ or average diastolic $\mathrm{BP}>89 \mathrm{mmHg}$ or taking prescribed antihypertensive medications, logistic analysis showed that the vegetarian categories related to hypertension in a similar fashion to that reported for BP (Table 6). Specifically, vegans, lacto-ovo vegetarians and partial vegetarians had lower estimated odds of hypertension $(\mathrm{OR}=0.37$ (95\% CI $0.19,0.74), \mathrm{OR}=0.57(95 \%$ CI $0.36,0.92)$ and $\mathrm{OR}=0.92(95 \%$ CI $0.50,1.70))$ than non-vegetarians and the odds ratios diminished substantially $(\mathrm{OR}=0.53$ (95\% CI $0.25,1 \cdot 11), \mathrm{OR}=0.86(95 \%$ CI $0.51,1 \cdot 45)$ and $\mathrm{OR}=1 \cdot 22(95 \%$ CI $0 \cdot 64,2 \cdot 33))$ when BMI was added to the model. This again suggests that the effect of diet to reduce $\mathrm{BP}$ is partly mediated by dietary effects on BMI.

Adding alcohol intake to any of the above models (not shown) changed results only trivially and the alcohol effect was always far from statistical significance. This is not surprising given the infrequent and small intakes.

In AHS-2, overnight urinary $\mathrm{K}$ (but not $\mathrm{Na}$ or $\mathrm{Ca}$ ) excretion correlates well with the corresponding dietary 
Table 6 Odds ratio and $95 \%$ confidence interval for hypertensiont by dietary pattern with and without BMI: white subjects representing the Adventist Health Study-2 (AHS-2) cohort, USA and Canada ( $n$ 500)

\begin{tabular}{|c|c|c|c|c|c|c|}
\hline \multirow[b]{2}{*}{ Effect } & \multicolumn{3}{|c|}{ OR estimates without BMI } & \multicolumn{3}{|c|}{ OR estimates with BMI } \\
\hline & Point estimate & $95 \% \mathrm{Cl}$ & $P$ value & Point estimate & $95 \% \mathrm{Cl}$ & $P$ value \\
\hline Non-vegetarian & 0.00 & & & 0.00 & & \\
\hline Partial vegetarian & 0.92 & $0 \cdot 50,1 \cdot 70$ & $0 \cdot 79$ & $1 \cdot 22$ & $0 \cdot 64,2 \cdot 33$ & 0.55 \\
\hline Lacto-ovo vegetarian & 0.57 & $0.36,0.92$ & 0.02 & $0 \cdot 86$ & $0 \cdot 51,1 \cdot 45$ & 0.57 \\
\hline Vegan & 0.37 & $0 \cdot 19,0 \cdot 74$ & 0.005 & 0.53 & $0 \cdot 25,1 \cdot 11$ & 0.09 \\
\hline Gender & 0.97 & $0.63,1.50$ & 0.90 & 0.99 & $0.63,1.55$ & 0.97 \\
\hline Age & $1 \cdot 07$ & $1 \cdot 05,1.09$ & $<0.0001$ & $1 \cdot 08$ & $1 \cdot 06,1 \cdot 10$ & $<0.0001$ \\
\hline Exercise & 0.99 & $0 \cdot 96,1 \cdot 02$ & 0.50 & $1 \cdot 00$ & $0.96,1.03$ & 0.89 \\
\hline \multicolumn{7}{|l|}{ BMI $\left(\mathrm{kg} / \mathrm{m}^{2}\right)$} \\
\hline$\leq 24.9$ & & & & 0.00 & & \\
\hline $25 \cdot 0-29 \cdot 9$ & & & & $1 \cdot 53$ & $0 \cdot 92,2 \cdot 53$ & 0.09 \\
\hline$\geq 30 \cdot 0$ & & & & $4 \cdot 64$ & $2 \cdot 66,8 \cdot 11$ & $<0.0001$ \\
\hline
\end{tabular}

tHypertension defined as average systolic blood pressure $>139 \mathrm{mmHg}$ or average diastolic blood pressure $>89 \mathrm{mmHg}$ or taking antihypertensive medications.

intake $(r=0.55$ for $\mathrm{K}$ compared with six dietary recalls; GE Fraser, unpublished results). Urinary $\mathrm{K}$ results were available from a random thirty-six calibration study subjects who were not taking antihypertensive medications. The latter were excluded as frequent use of diuretics will distort results. The overnight $\mathrm{K}$ excretion in vegetarians (vegans and lacto-ovo vegetarians combined) was on average $30 \cdot 0 \mathrm{mmol} / \mathrm{l}$ and $19 \cdot 8 \mathrm{mmol} / 1$ for non-vegetarians $(P=0 \cdot 10)$. Although compatible with chance, this suggests a sizeable difference in $\mathrm{K}$ intake as would be expected.

\section{Discussion}

In this non-black population we report significant differences in both systolic and diastolic BP and the odds of hypertension, depending on vegetarian dietary pattern. These results are from a population that includes a wide variety of age, socio-economic status and both genders, and they represent dietary habits stable over many years for the most part. Subjects summarized their diets over the previous year. Few other studies have been able to compare habitual non-vegetarians with both habitual vegans and lacto-ovo vegetarians. The effect appears to be moderately stronger in vegans as there were fewer vegans taking antihypertensive medications and those not taking such medications had BP as low (approximately) as the lacto-ovo vegetarians. Effects appear to be partially explained by dietary effects on BMI, which are strong in this population ${ }^{(36)}$. Dietary effects were still evident in those with normal BMI.

The percentage overweight or obese in this population was lower than in the general US population ${ }^{(36)}$, though still considerable. Our results are adjusted for these factors but it seems likely that the effect of a vegetarian diet to reduce body weight is one mechanism partially responsible for the BP effect. However, some additional effect probably still remains. A partial mediating effect of BMI is consistent with the results of some other observational studies ${ }^{(21,27)}$. Interestingly, in previous short-term feeding studies there was typically no weight loss during the vegetarian feeding period, despite the well-known long-term large differences in BMI between vegetarians and others. It does seem possible that although no weight changes were seen over a few weeks of the feeding studies, physiological processes (e.g. insulin/glucose metabolism) ${ }^{(19)}$ resulting in or associated with weight loss over a longer period may have begun and may already have started to affect BP.

As with most other similar studies, we found effects on both systolic and diastolic BP. While a few reported studies found changes in systolic ${ }^{(9,20,22)}$ pressures only, this may have been due to limited statistical power.

Beyond BMI, which dietary factors in the vegetarian diet may account for effects on BP levels is not well understood. Vegetarians have higher fibre and $\mathrm{K}$ intakes as a result of their greater intakes of fruits, vegetables, fruits, nuts and wholegrain products ${ }^{(37-39)}$. Recent metaanalyses of randomized trials ${ }^{(16,17)}$ demonstrate small but significant effects of fibre, particularly in those with higher baseline pressures.

Consumption of a K-rich diet has a natriuretic effect and diets that are high in $\mathrm{K}$ usually are low in $\mathrm{Na}$, as long as unprocessed foods are consumed ${ }^{(5)}$. Low Ca or dairy intake has also been associated with higher $\mathrm{BP}^{(40-42)}$. Inconsistent with this, our results and those from the European Prospective Investigation into Cancer and Nutrition (EPIC)-Oxford study ${ }^{(27)}$ show that vegans who avoid dairy products have the least hypertension. However, Adventist vegans do not have particularly low Ca intakes, perhaps due in part to supplementation but also because of vegetable sources of $\mathrm{Ca}$.

Proposed physiological mechanisms that may mediate the effect of a vegetarian diet include modulation of baroreceptor sensitivity, direct vasodilatory effects, changes in catecholamine and renin-angiotensin-aldosterone metabolism, improvement of glucose tolerance with lower insulin levels ${ }^{(5,19)}$, and lower blood viscosity in vegetarians ${ }^{(43)}$. 


\section{Strengths and weaknesses}

We measured BP using an automated sphygmomanometer which provides BP with acceptable validity ${ }^{(30)}$. A large amount of apparently random error is associated with BP measurement, despite taking measures to counteract the known influencing factors. In addition to using the digital machine we standardized other environmental factors that may influence BP. Together with the relatively large number of study subjects these factors should reduce the effects of random errors. Most study subjects had been church members for decades, further suggesting stability of dietary habits. Differences in duration of church membership by dietary category seem unlikely to affect results given that only $8-21 \%$ had been members for $<25$ years across the dietary groups.

It was necessary to assign vegetarian dietary pattern based on the results of an FFQ rather than the repeated recalls, as a small number of recalls will easily miss less frequent consumption of animal products. There is inevitably recall and reporting error in these data. However, compared with the average of six $24 \mathrm{~h}$ recalls, correlation coefficients $(r)$ corrected for deattenuation are as follows: red meat $(r=0 \cdot 76)$; poultry $(r=0 \cdot 76)$; fish $(r=0 \cdot 53)$; eggs $(r=0 \cdot 64)$; dairy protein $(r=0 \cdot 77)$. Thus by usual standards the validity of food frequency questions used in the algorithm assigning vegetarian status is excellent.

Inevitably there were small amounts of missing dietary data $(<1 \%$ for most composite variables in this study). However, $9 \cdot 4 \%$ of subjects were missing at least one of the longer lists of individual dairy items where we needed to assume missing at random for imputation and this may have resulted in a small amount of misclassification.

The proportion of this older study population who were taking antihypertensive medication was $25 \cdot 3 \%$, compared with $21 \cdot 3 \%$ in the US population aged 18 years and above ${ }^{(44)}$. It is interesting that $10.0 \%$ of vegans but $28.8 \%$ of non-vegetarians are taking antihypertensive medications. This could mean that the vegan population is less willing to take medications or that $\mathrm{BP}$ is indeed lower in this subgroup. Given the relatively low BP among vegans not taking antihypertensive medications, their need for medications is probably less. The much lower odds of hypertension in this group is thus due both to the lower proportion taking antihypertensive medication and the low $\mathrm{BP}$ in those not taking medication.

As this is a cross-sectional study we do not know the stability of dietary patterns over time, meaning that it is not possible to exclude a reverse causation in that some may have changed their diet after they received a diagnosis of elevated BP. However, if this occurred it would likely work against reverse causation as most Adventists with a health problem would actually move towards a plant-based diet.

\section{Conclusions}

Our study extends and supports previous evidence that diet affects measured BP levels, both systolic and diastolic, with vegans and lacto-ovo vegetarians having lower BP than non-vegetarians. We show that this appears to be long-lasting as our subjects generally have maintained these dietary habits over at least 1 year. The vegans appear to have the least hypertension, although further evidence should be gathered on this group. Our data represent a diverse population (although all Adventists) by geography, BMI, socio-economic status and gender. Many Americans may benefit from a diet containing more plant foods to prevent hypertension.

\section{Acknowledgements}

This study was funded by a grant from the National Cancer Institute, National Institutes of Health (\# 5RO1 CA 094594). There are no conflicts of interest to disclose. B.J.P. drafted the manuscript. R.A. wrote the section on statistical analyses, and with J.F. performed the statistical analyses. K.J.-S. and G.E.F. helped collect the data and conceived the study. B.J.P., K.J.-S. and G.E.F. were all involved in study design. All authors reviewed the manuscript on several occasions.

\section{References}

1. World Health Organization (2002) The World Health Report 2002: Reducing Risks, Promoting Healthy Life. Geneva: WHO.

2. National Center for Health Statistics (2010) Health, United States, 2009: With Special Feature on Medical Technology. Hyattsville, MD: NCHS.

3. Schoenborn CA \& Heyman KM (2009) Health characteristics of adults aged 55 years and over: United States, 2004-2007. Natl Health Stat Report issue 16, 1-31.

4. Terry DF, Pencina MJ, Vasan RS et al. (2005) Cardiovascular risk factors predictive for survival and morbidity-free survival in the oldest-old Framingham Heart Study participants. J Am Geriatr Soc 53, 1944-1950.

5. Suter PM, Sierro C \& Vetter W (2002) Nutritional factors in the control of blood pressure and hypertension. Nutr Clin Care 5, 9-19.

6. Berkow SE \& Barnard ND (2005) Blood pressure regulation and vegetarian diets. Nutr Rev 63, 1-8.

7. Nowson CA, Wattanapenpaiboon N \& Pachett A (2009) Low-sodium Dietary Approaches to Stop Hypertensiontype diet including lean red meat lowers blood pressure in postmenopausal women. Nutr Res 29, 8-18.

8. Hodgson JM, Burke V, Beilin LJ et al. (2006) Partial substitution of carbohydrate intake with protein intake from lean red meat lowers blood pressure in hypertensive persons. Am J Clin Nutr 83, 780-787.

9. Sacks FM, Donner A, Castelli WP et al. (1981) Effect of ingestion of meat on plasma cholesterol of vegetarians. JAMA 246, 640-644.

10. Iacono JM, Judd JT, Marshall MW et al. (1981) The role of dietary essential fatty acids and prostaglandins in reducing blood pressure. Prog Lipid Res 20, 349-364. 
11. Iacono JM, Puska P, Dougherty RM et al. (1983) Effect of dietary fat on blood pressure in a rural Finnish population. Am J Clin Nutr 38, 860-869.

12. Margetts BM, Beilin LJ, Armstrong BK et al. (1984) Dietary fats and blood pressure. Aust NZ J Med 14, 444-447.

13. Margetts BM, Beilin LJ, Armstrong BK et al. (1988) Vegetarian diet in mild hypertension: effects of fat and fiber. Am J Clin Nutr 48, 801-805.

14. Puska P, Iacono JM, Nissinen A et al. (1985) Dietary fat and blood pressure: an intervention study on the effects of a low-fat diet with two levels of polyunsaturated fat. Prev Med 14, 573-584.

15. Sacks FM, Marais GE, Handysides G et al. (1984) Lack of an effect of dietary saturated fat and cholesterol on blood pressure in normotensives. Hypertension 6, 193-198.

16. Streppel MT, Arends LR, van 't Veer P et al. (2005) Dietary fiber and blood pressure: a meta-analysis of randomized placebo-controlled trials. Arch Intern Med 165, 150-156.

17. Whelton SP, Hyre AD, Pedersen B et al. (2005) Effect of dietary fiber intake on blood pressure: a meta-analysis of randomized, controlled clinical trials. J Hypertens $\mathbf{2 3}$, 475-481.

18. Rouse IL, Beilin LJ, Mahoney DP et al. (1983) Vegetarian diet and blood pressure. Lancet 2, 742-743.

19. Sciarrone SE, Strahan MT, Beilin LJ et al. (1993) Biochemical and neurohormonal responses to the introduction of a lacto-ovovegetarian diet. J Hypertens 11, 849-860.

20. Margetts BM, Beilin LJ, Vandongen R et al. (1986) Vegetarian diet in mild hypertension: a randomised controlled trial. Br Med J (Clin Res Ed) 293, 1468-1471.

21. Rouse IL, Armstrong BK \& Beilin LJ (1983) The relationship of blood pressure to diet and lifestyle in two religious populations. J Hypertens 1, 65-71.

22. Armstrong B, Clarke H, Martin C et al. (1979) Urinary sodium and blood pressure in vegetarians. Am J Clin Nutr 32, 2472-2476.

23. Ophir O, Peer G, Gilad J et al. (1983) Low blood pressure in vegetarians: the possible role of potassium. Am J Clin Nutr 37, 755-762.

24. Burr ML, Bates CJ, Fehily AM et al. (1981) Plasma cholesterol and blood pressure in vegetarians. J Hum Nutr 35, 437-441.

25. Melby CL, Goldflies DG \& Toohey ML (1993) Blood pressure differences in older black and white long-term vegetarians and nonvegetarians. J Am Coll Nutr 12, 262-269.

26. Melby CL, Toohey ML \& Cebrick J (1994) Blood pressure and blood lipids among vegetarian, semivegetarian, and nonvegetarian African Americans. Am J Clin Nutr 59, 103-109.

27. Appleby PN, Davey GK \& Key TJ (2002) Hypertension and blood pressure among meat eaters, fish eaters, vegetarians and vegans in EPIC-Oxford. Public Health Nutr 5, 645-654.

28. Butler TL, Fraser GE, Beeson WL et al. (2008) Cohort profile: The Adventist Health Study-2 (AHS-2). Int $J$ Epidemiol 37, 260-265.
29. Chan J, Knutsen SF, Sabate J et al. (2007) Feasibility of running clinics to collect biological specimens in a nationwide cohort study - Adventist Health Study-2. Ann Epidemiol 17, 454-457.

30. O'Brien E, Mee F, Atkins N et al. (1996) Evaluation of three devices for self-measurement of blood pressure according to the revised British Hypertension Society Protocol: the Omron HEM-705CP, Philips HP5332, and Nissei DS-175. Blood Press Monit 1, 55-61.

31. Jaceldo-Siegl K, Knutsen SF, Sabate J et al. (2010) Validation of nutrient intake using an FFQ and repeated $24 \mathrm{~h}$ recalls in black and white subjects of the Adventist Health Study-2 (AHS-2). Public Health Nutr 13, 812-819.

32. Jaceldo-Siegl K, Fan J, Sabate J et al. (2011) Race-specific validation of food intake obtained from a comprehensive FFQ: the Adventist Health Study-2. Public Health Nutr 14, 1988-1997.

33. Fraser G \& Yan R (2007) Guided multiple imputation of missing data: using a subsample to strengthen the missingat-random assumption. Epidemiology 18, 246-252.

34. R DCT (2010) $R: A$ Language and Environment for Statistical Computing, Version 2.11.1 (2010-05-31). Vienna: R Foundation for Statistical Computing.

35. Sallis JF, Haskell WL, Wood PD et al. (1985) Physical activity assessment methodology in the Five-City Project. Am J Epidemiol 121, 91-106.

36. Tonstad S, Butler T, Yan R et al. (2009) Type of vegetarian diet, body weight, and prevalence of type 2 diabetes. Diabetes Care 32, 791-796.

37. Haddad EH \& Tanzman JS (2003) What do vegetarians in the United States eat? Am J Clin Nutr 78, 3 Suppl., 626S-632S

38. Larsson CL \& Johansson GK (2002) Dietary intake and nutritional status of young vegans and omnivores in Sweden. Am J Clin Nutr 76, 100-106.

39. Janelle KC \& Barr SI (1995) Nutrient intakes and eating behavior scores of vegetarian and nonvegetarian women. J Am Diet Assoc 95, 180-186.

40. Ruidavets JB, Bongard V, Simon C et al. (2006) Independent contribution of dairy products and calcium intake to blood pressure variations at a population level. J Hypertens 24, 671-681.

41. McCarron DA \& Reusser ME (1999) Finding consensus in the dietary calcium-blood pressure debate. J Am Coll Nutr 18, 5 Suppl., 398S-405S.

42. McCarron DA \& Reusser ME (2001) Are low intakes of calcium and potassium important causes of cardiovascular disease? Am J Hypertens 14, 6 Pt 2, 206S-212S.

43. Ernst E, Pietsch L, Matrai A et al. (1986) Blood rheology in vegetarians. Br J Nutr 56, 555-560.

44. Ostchega Y, Yoon SS, Hughes J et al. (2008) Hypertension awareness, treatment, and control - continued disparities in adults: United States, 2005-2006. NCHS Data Brief issue $3,1-8$ 